\title{
Multi-particle eccentricities in collisions dominated by fluctuations
}

\author{
Adam Bzdak, Vladimir Skokov
}

Submitted to Nuclear Physics A

August 2015

Physics Department

Brookhaven National Laboratory

\author{
U.S. Department of Energy \\ USDOE Office of Science (SC), \\ Nuclear Physics (NP) (SC-26)
}

Notice: This manuscript has been authored by employees of Brookhaven Science Associates, LLC under Contract No. DE- SC0012704 with the U.S. Department of Energy. The publisher by accepting the manuscript for publication acknowledges that the United States Government retains a non-exclusive, paid-up, irrevocable, world-wide license to publish or reproduce the published form of this manuscript, or allow others to do so, for United States Government purposes. 


\section{DISCLAIMER}

This report was prepared as an account of work sponsored by an agency of the United States Government. Neither the United States Government nor any agency thereof, nor any of their employees, nor any of their contractors, subcontractors, or their employees, makes any warranty, express or implied, or assumes any legal liability or responsibility for the accuracy, completeness, or any third party's use or the results of such use of any information, apparatus, product, or process disclosed, or represents that its use would not infringe privately owned rights. Reference herein to any specific commercial product, process, or service by trade name, trademark, manufacturer, or otherwise, does not necessarily constitute or imply its endorsement, recommendation, or favoring by the United States Government or any agency thereof or its contractors or subcontractors. The views and opinions of authors expressed herein do not necessarily state or reflect those of the United States Government or any agency thereof. 
BNL-112081-2016-JA

\title{
Multi-particle eccentricities in collisions dominated by fluctuations
}

\author{
Adam Bzdak $^{\mathrm{a}, \mathrm{b}, *}$, Vladimir Skokov ${ }^{\mathrm{b}, \mathrm{c}, *}$ \\ ${ }^{a}$ AGH University of Science and Technology, Faculty of Physics and Applied Computer Science, \\ 30-059 Kraków, Poland \\ b RIKEN BNL Research Center, Brookhaven National Laboratory, Upton, NY 11973, USA \\ ${ }^{c}$ Department of Physics, Western Michigan University, Kalamazoo, MI 49008, USA
}

\begin{abstract}
We compute analytically the multi-particle eccentricities, $\epsilon_{m}\{2 n\}$, for systems dominated by fluctuations, such as proton-nucleus collisions at the Large Hadron Collider. In particular, we derive a general relation for $\left\langle\epsilon_{2}^{2 n}\right\rangle$. We further discuss the relations between various multi-particle eccentricities and demonstrate that $\epsilon_{2}\{2\}>\epsilon_{2}\{4\} \simeq \epsilon_{2}\{6\} \simeq \epsilon_{2}\{8\}$, in agreement with recent numerical calculations in a Glauber model. (c) 2015 Elsevier B.V. All rights reserved.
\end{abstract}

Keywords: LHC; p + A; Quark-gluon plasma

\section{Introduction}

Recent measurements of high multiplicity proton-proton $(p+p)$ and proton-nucleus $(p+A)$ collisions at the Large Hadron Collider (LHC) revealed an unexpected enhancement of the twoparticle correlation function at small azimuthal angles and large separation in rapidity [1-4]. This effect was also seen at the Relativistic Heavy Ion Collider (RHIC) in deuteron-gold ( $+\mathrm{Au}$ ) and helium-gold $\left({ }^{3} \mathrm{He}+\mathrm{Au}\right)$ collisions $[5,6]$.

\footnotetext{
* Corresponding authors.

E-mail addresses: bzdak@ fis.agh.edu.pl (A. Bzdak), vskokov@ quark.phy.bnl.gov (V. Skokov).
} 
The same correlation pattern was previously observed in nucleus-nucleus $(\mathrm{A}+\mathrm{A})$ collisions at RHIC [7]. In nucleus-nucleus collisions the azimuthal angle correlation function and its Fourier harmonics are well described by the relativistic hydrodynamics, an effective theory of long wave excitations in a strongly coupled system [8]. Using viscous hydrodynamics, the ratio of the shear viscosity over the entropy is found to be surprisingly small (see, e.g., Ref. [9]) and close to the conjectured lowest bound for a strongly interacting system [10].

Nucleus-nucleus collisions are immensely complicated, due to multi-particle rescattering, the possible formation of a thermal system, and subsequent collective evolution. They do not offer a direct possibility to study initial state effects. It was expected that elementary $\mathrm{p}+\mathrm{p}$ and $\mathrm{p}+\mathrm{A}$ collisions are dominated by the initial state effects and thus their behavior can be studied and described by quantum chromodynamics (QCD) at weak coupling. However, due to the high densities of partons, the effects of gluon saturation must be taken into account. This is done in the framework of the Color Glass Condensate (CGC) [11], an effective description of a hadron at asymptotically high energy in the regime of weakly coupled QCD. Although at present there is no compelling experimental evidence indicating that the $\mathrm{CGC}$ is an appropriate tool to interpret hadronic collisions at the LHC energies, there are attempts to describe the azimuthal angle correlation functions in $\mathrm{p}+\mathrm{p}$ and $\mathrm{p}+\mathrm{A}$ collisions at the LHC [12], see also Refs. [13-15], and very recent development in Ref. [16], which in particular showed that conventional CGC used in Ref. [12] is incompatible with the experimental data at high multiplicity. Recently, hydrodynamics was applied to $\mathrm{p}+\mathrm{A}$ collisions [17-22] with a reasonably good fit to the data. This success however does not solve several conceptual theoretical problems, such as how quickly thermalization occurs, whether the initial conditions are boost invariant, and many other effects, which are implicitly assumed when hydrodynamics is applied. Very recently some of these problems were addressed in the AdS/CFT framework in Refs. [23,24]. Finally, a multi-phase transport model (AMPT) [25] was recently compared with the experimental data in p + p [26], p + A [27] and $\mathrm{d}+\mathrm{Au}$ [28] interactions. Possible origin of the anisotropies within the AMPT model are discussed in Ref. [29].

In summary, at present we have two general approaches for the high multiplicity $\mathrm{p}+\mathrm{p}$ and $\mathrm{p}+\mathrm{A}$ collisions at the LHC energy: models of strongly interacting medium (hydrodynamics, AdS/CFT, cascade) and a rival effective theory of QCD at high energies in the weakly interacting regime, the CGC. Several observables and ideas were recently put forward to single out an appropriate language to describe phenomena in these collisions [30-33].

The motivation for this short note is the observation of Ref. [34], where the authors showed that the initial eccentricities of the interaction region in $\mathrm{p}+\mathrm{A}$ and $\mathrm{A}+\mathrm{A}$ interactions form a peculiar hierarchy, namely, the eccentricities computed with the two and higher number of particles satisfy the following relation:

$$
\epsilon_{2}\{2\}>\epsilon_{2}\{4\} \simeq \epsilon_{2}\{6\} \simeq \epsilon_{2}\{8\} \simeq \ldots
$$

This relation was also verified in Ref. [35], where its origin was attributed to a power law distribution of $\epsilon_{2}$.

Equation (1) has serious phenomenological implications. First, if the same hierarchy is observed for the Fourier coefficients of the azimuthal correlation function in $\mathrm{p}+\mathrm{A}$ collisions, it would indicate that the azimuthal correlation of hadrons is determined by the geometry of the initial state. This favors approaches where the initial geometry is translated into momentum space from collective effects, such as in hydrodynamics. Current treatments of the CGC are independent of the geometry, so that equivalent hierarchy for the Fourier coefficients of the azimuthal correlation function is not apparent. 
In this paper, we extend the numerical results of Ref. [34] by analytically computing various eccentricities in a system dominated by fluctuations, e.g., $p+$ A. We provide compact analytical expressions that can be used for further analysis.

\section{Derivation of analytical results for eccentricities}

Suppose we distribute $N$ points on a plane. Let the distribution be $P(r, \phi)$, where $r$ and $\phi$ are the polar coordinates of the points. ${ }^{1}$ The ellipticity squared in a given event is defined as $[36,37]$

$$
\epsilon_{2}^{2}=\frac{\left[\sum_{i=1}^{N} r_{i}^{2} \cos \left(2 \phi_{i}\right)\right]^{2}+\left[\sum_{i=1}^{N} r_{i}^{2} \sin \left(2 \phi_{i}\right)\right]^{2}}{\left(\sum_{i=1}^{N} r_{i}^{2}\right)^{2}}=\frac{\left(\sum_{k=1}^{N} r_{k}^{2} e^{i 2 \phi_{k}}\right)\left(\sum_{l=1}^{N} r_{l}^{2} e^{-i 2 \phi_{l}}\right)}{\left(\sum_{i=1}^{N} r_{i}^{2}\right)^{2}}
$$

The goal of this brief note is to calculate $\left\langle\epsilon_{2}^{2 n}\right\rangle$ analytically for an arbitrary value of $n$, where $\langle\ldots\rangle$ denotes the average over many events. All our results can be immediately generalized to, e.g., triangularity by replacing $r^{2} \rightarrow r^{3}$, etc. ${ }^{2}$ In this calculation we make two assumptions which, as we argue below, are well justified. First, calculating Eq. (2) we assume that $\langle A / B\rangle=\langle A\rangle /\langle B\rangle .^{3}$ In this case

$$
\left\langle\epsilon_{2}^{2 n}\right\rangle \equiv \frac{\left\langle\epsilon_{2}^{2 n}\right\rangle_{\mathrm{num}}}{\left\langle\epsilon_{2}^{2 n}\right\rangle_{\mathrm{deno}}}=\frac{\left\langle\left(\sum_{k=1}^{N} r_{k}^{2} e^{i 2 \phi_{k}}\right)^{n}\left(\sum_{l=1}^{N} r_{l}^{2} e^{-i 2 \phi_{l}}\right)^{n}\right\rangle}{\left\langle\left(\sum_{i=1}^{N} r_{i}^{2}\right)^{2 n}\right\rangle}
$$

To simplify our notation we introduce $\left\langle\epsilon_{2}^{2 n}\right\rangle_{\text {num }}$ and $\left\langle\epsilon_{2}^{2 n}\right\rangle_{\text {deno }}$ to denote the numerator and the denominator of Eq. (3).

In the following we neglect the recentering correction, i.e., the coordinate system is not shifted to the center of mass. We expect this correction to modify slightly our results only for small $N$. We will come back to this point later in this Section, were we compare our analytical calculations with Monte Carlo (MC) simulations.

To simplify equations we introduce the following notation

$$
\begin{aligned}
\mathcal{D} r & =d r_{1} \ldots d r_{N} r_{1} \ldots r_{N}, \\
\mathcal{D} \phi & =d \phi_{1} \ldots d \phi_{N}, \\
\mathcal{P}(r, \phi) & =P\left(r_{1}, \phi_{1}\right) \ldots P\left(r_{N}, \phi_{N}\right),
\end{aligned}
$$

where in the last equation we explicitly assume that all the points are sampled independently. The average over many events is thus

$$
\langle A\rangle=\int \mathcal{D} r \mathcal{D} \phi \mathcal{P}(r, \phi) A .
$$

The denominator can be straightforwardly computed:

\footnotetext{
1 The distribution $P(r, \phi)$ is normalized to unity.

2 This depends on the definition of triangularity and higher eccentricities. If we define them with $r^{2}$ than our results hold for all eccentricities.

3 We found that this approximation reproduces the exact result with a Gaussian distribution $P(r)$, see Ref. [35].
} 


$$
\begin{aligned}
\left\langle\epsilon_{2}^{2 n}\right\rangle_{\mathrm{deno}} & =\int \mathcal{D} r \mathcal{D} \phi \mathcal{P}(r, \phi)\left(\sum_{i=1}^{N} r_{i}^{2}\right)^{2 n} \\
& =\int \mathcal{D} r \mathcal{D} \phi \mathcal{P}(r, \phi) \lim _{x \rightarrow 0} \frac{d^{2 n}}{d x^{2 n}} e^{-x \sum_{i=1}^{N} r_{i}^{2}} \\
& =\lim _{x \rightarrow 0} \frac{d^{2 n}}{d x^{2 n}}\left(\int d \phi r d r P(r, \phi) e^{-x r^{2}}\right)^{N} \\
& =\lim _{x \rightarrow 0} \frac{d^{2 n}}{d x^{2 n}}\left(\int r d r P_{r}(r) e^{-x r^{2}}\right)^{N} \\
& =\lim _{x \rightarrow 0} \frac{d^{2 n}}{d x^{2 n}}\left\langle e^{-x r^{2}}\right\rangle^{N} .
\end{aligned}
$$

It is worth emphasizing that Eq. (8) is valid for an arbitrary $P(r, \phi)$.

Following the same procedure, the numerator of Eq. (3) can be written in the form

$$
\begin{aligned}
\left\langle\epsilon_{2}^{2 n}\right\rangle_{\text {num }}= & \int \mathcal{D} r \mathcal{D} \phi \mathcal{P}(r, \phi)\left(\sum_{k=1}^{N} r_{k}^{2} e^{i 2 \phi_{k}}\right)^{n}\left(\sum_{l=1}^{N} r_{l}^{2} e^{-i 2 \phi_{l}}\right)^{n} \\
= & \int \mathcal{D} r \mathcal{D} \phi \mathcal{P}(r, \phi) \lim _{x \rightarrow 0} \frac{d^{n}}{d x^{n}} \lim _{y \rightarrow 0} \frac{d^{n}}{d y^{n}} \\
& \times \exp \left(-x \sum_{k=1}^{N} r_{k}^{2} e^{i 2 \phi_{k}}-y \sum_{l=1}^{N} r_{l}^{2} e^{-i 2 \phi_{l}}\right) \\
= & \lim _{x \rightarrow 0} \frac{d^{n}}{d x^{n}} \lim _{y \rightarrow 0} \frac{d^{n}}{d y^{n}}\left(\int d \phi r d r P(r, \phi) \exp \left(-x r^{2} e^{i 2 \phi}-y r^{2} e^{-i 2 \phi}\right)\right)^{N} \\
= & \lim _{x \rightarrow 0} \frac{d^{n}}{d x^{n}} \lim _{y \rightarrow 0} \frac{d^{n}}{d y^{n}}\left\langle\exp \left(-x r^{2} e^{i 2 \phi}\right) \exp \left(-y r^{2} e^{-i 2 \phi}\right)\right\rangle^{N} .
\end{aligned}
$$

This equation can be used to express $\left\langle\epsilon_{2}^{2 n}\right\rangle_{\text {num }}$ through $\left\langle r^{k} e^{i m \phi}\right\rangle$ for a general function $P(r, \phi) .^{4}$ Similar problem was extensively studied in the literature up to $n=2$ [38,39]. Our Eq. (9) allows to derive an exact relation for an arbitrary value of $n$. However, in the present paper we are only interested in the system dominated by fluctuations, that is, we neglect the $\phi$ dependence in $P(r, \phi)$ and assume that $P(r, \phi)=P_{r}(r)$. Expanding exponents in Eq. (9) we obtain

$$
\begin{aligned}
\left\langle\epsilon_{2}^{2 n}\right\rangle_{\text {num }} & =\lim _{x \rightarrow 0} \frac{d^{n}}{d x^{n}} \lim _{y \rightarrow 0} \frac{d^{n}}{d y^{n}}\left\langle\sum_{k=0}^{\infty} \sum_{l=0}^{\infty} \frac{\left(-x r^{2}\right)^{k}\left(-y r^{2}\right)^{l}}{k ! l !} e^{i 2 \phi(k-l)}\right\rangle^{N} \\
& =\lim _{x \rightarrow 0} \frac{d^{n}}{d x^{n}} \lim _{y \rightarrow 0} \frac{d^{n}}{d y^{n}}\left\langle\sum_{k=0}^{\infty} \frac{\left(x y r^{4}\right)^{k}}{k ! k !}\right\rangle^{N} \\
& =\lim _{x \rightarrow 0} \frac{d^{n}}{d x^{n}} \lim _{y \rightarrow 0} \frac{d^{n}}{d y^{n}}\left\langle I_{0}\left(2 r^{2} \sqrt{x y}\right)\right\rangle^{N} \\
& =\lim _{x \rightarrow 0} \frac{d^{n}}{d x^{n}} \lim _{z \rightarrow 0} x^{n} \frac{d^{n}}{d z^{n}}\left\langle I_{0}\left(2 r^{2} \sqrt{z}\right)\right\rangle^{N} \\
& =n ! \lim _{z \rightarrow 0} \frac{d^{n}}{d z^{n}}\left\langle I_{0}\left(2 r^{2} \sqrt{z}\right)\right\rangle^{N}
\end{aligned}
$$

${ }^{4}$ For example, $\left\langle\epsilon_{2}^{2}\right\rangle_{\text {num }}=N(N-1)\left\langle r^{2} e^{i 2 \phi}\right\rangle\left\langle r^{2} e^{-i 2 \phi}\right\rangle+N\left\langle r^{4}\right\rangle$. 
where $I_{0}(x)$ is the modified Bessel function. Again, Eq. (10) can be used to derive a relation between $\left\langle\epsilon_{2}^{2 n}\right\rangle_{\text {num }}$ and $\left\langle r^{k}\right\rangle$. We will present appropriate expressions in Section 3.

The final result of our computations is given by

$$
\left\langle\epsilon_{2}^{2 n}\right\rangle=\frac{n ! \lim _{z \rightarrow 0} \frac{d^{n}}{d z^{n}}\left\langle I_{0}\left(2 \sqrt{z} r^{2}\right)\right\rangle^{N}}{\lim _{z \rightarrow 0} \frac{d^{2 n}}{d z^{2 n}}\left\langle e^{-r^{2} z}\right\rangle^{N}} .
$$

It would be interesting and pedagogical to demonstrate the effectiveness of this result for a simple case of a Gaussian distribution.

\subsection{Gaussian distribution}

Equation (11) can be computed analytically for a normalized Gaussian distribution, $P_{r}(r)=$ $2 e^{-r^{2} / \sigma^{2}} / \sigma^{2}$. In this case

$$
\left\langle e^{-r^{2} z}\right\rangle=2 \int_{0}^{\infty} \frac{r d r}{\sigma^{2}} e^{-r^{2} / \sigma^{2}} e^{-r^{2} z}=\frac{1}{1+z \sigma^{2}}
$$

and

$$
\left\langle I_{0}\left(2 r^{2} \sqrt{z}\right)\right\rangle=2 \int_{0}^{\infty} \frac{r d r}{\sigma^{2}} e^{-r^{2} / \sigma^{2}} I_{0}\left(2 r^{2} \sqrt{z}\right)=\frac{1}{\sqrt{1-4 z \sigma^{4}}} .
$$

Substituting into Eq. (11) and differentiating with respect to $z$ we obtain

$$
\begin{aligned}
& \left\langle\epsilon_{2}^{2 n}\right\rangle_{\text {num }}=n ! 4^{n} \sigma^{4 n} \frac{N}{2}\left(\frac{N}{2}+1\right) \ldots\left(\frac{N}{2}+n-1\right), \\
& \left\langle\epsilon_{2}^{2 n}\right\rangle_{\text {deno }}=\sigma^{4 n} N(N+1) \ldots(N+2 n-1)
\end{aligned}
$$

and finally

$$
\left\langle\epsilon_{2}^{2 n}\right\rangle=\frac{n ! 4^{n} \frac{N}{2}\left(\frac{N}{2}+1\right) \ldots\left(\frac{N}{2}+n-1\right)}{N(N+1) \ldots(N+2 n-1)} .
$$

As expected $\left\langle\epsilon_{2}^{2 n}\right\rangle$ does not depend on the width of the distribution, $\sigma$. Using Eq. (16) various cumulants $[40,41]$ can be also straightforwardly computed for a Gaussian distribution in $r$. For example

$$
\begin{aligned}
\epsilon_{2}^{2}\{2\} & =\frac{2}{1+N}, \\
\epsilon_{2}^{4}\{4\} & =\frac{16}{(1+N)^{2}(3+N)}, \\
\epsilon_{2}^{6}\{6\} & =\frac{192}{(1+N)^{3}(3+N)(5+N)}, \\
\epsilon_{2}^{8}\{8\} & =\frac{6144(17+5 N)}{11(1+N)^{4}(3+N)^{2}(5+N)(7+N)} .
\end{aligned}
$$




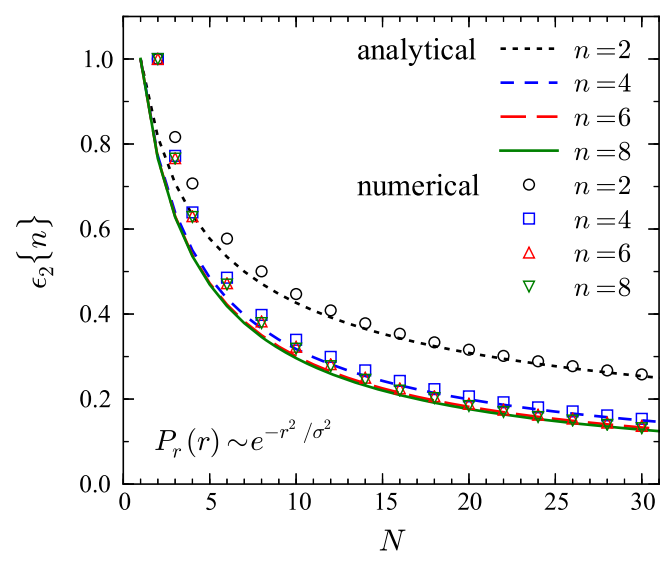

Fig. 1. The $n$-particle ellipticities $\epsilon_{2}\{n\}, n=2,4,6,8$, for various numbers of independent points, $N$, calculated analytically, Eqs. (17)-(20), compared with the Monte Carlo (MC) calculations (open symbols). As we checked, the difference between MC and Eqs. (17)-(20) comes solely from the recentering correction which we neglect in our analytical calculations.

Equations (17)-(20) agree with those obtained in Ref. [35]. It is easy to verify that

$$
\epsilon_{2}\{2\}>\epsilon_{2}\{4\} \approx \epsilon_{2}\{6\} \approx \epsilon_{2}\{8\}
$$

in agreement with the recent numerical calculations of Ref. [34]. In Fig. 1 we compare our analytical results with the full Monte Carlo calculations. We checked that the small deviation from the numerical results at small $N$ comes solely from the recentering ${ }^{5}$ correction that is neglected in our analytical calculations. ${ }^{6}$

We also performed calculations for different functions, e.g., $P_{r}(r)=e^{-r^{k} / \sigma^{k}}, k>2$, as discussed in the next section.

\section{General relations}

It is not always possible to calculate analytically $\left\langle I_{0}\left(2 r^{2} \sqrt{z}\right)\right\rangle$ and $\left\langle e^{-r^{2} z}\right\rangle$, thus in this section we derive general relations between $\left\langle\epsilon_{2}^{2 n}\right\rangle$ and $\left\langle r^{m}\right\rangle$ for an arbitrary function $P_{r}(r)$. Taking derivatives of Eq. (8) and Eq. (10) (second line) we obtain $\left[N_{n} \equiv \frac{N !}{(N-n) !}\right.$ and $\left.N \equiv N_{1}\right]$

$$
\begin{aligned}
\left\langle\epsilon_{2}^{2}\right\rangle & =\frac{N\left\langle r^{4}\right\rangle}{N_{2}\left\langle r^{2}\right\rangle^{2}+N\left\langle r^{4}\right\rangle}, \\
\left\langle\epsilon_{2}^{4}\right\rangle_{\text {num }} & =2 N_{2}\left\langle r^{4}\right\rangle^{2}+N\left\langle r^{8}\right\rangle, \\
\left\langle\epsilon_{2}^{4}\right\rangle_{\text {deno }} & =N_{4}\left\langle r^{2}\right\rangle^{4}+6 N_{3}\left\langle r^{2}\right\rangle^{2}\left\langle r^{4}\right\rangle+3 N_{2}\left\langle r^{4}\right\rangle^{2}+4 N_{2}\left\langle r^{2}\right\rangle\left\langle r^{6}\right\rangle+N\left\langle r^{8}\right\rangle, \\
\left\langle\epsilon_{2}^{6}\right\rangle_{\text {num }} & =6 N_{3}\left\langle r^{4}\right\rangle^{3}+9 N_{2}\left\langle r^{4}\right\rangle\left\langle r^{8}\right\rangle+N\left\langle r^{12}\right\rangle, \\
\left\langle\epsilon_{2}^{6}\right\rangle_{\text {deno }} & =N_{6}\left\langle r^{2}\right\rangle^{6}+15 N_{5}\left\langle r^{2}\right\rangle^{4}\left\langle r^{4}\right\rangle+45 N_{4}\left\langle r^{2}\right\rangle^{2}\left\langle r^{4}\right\rangle^{2}+15 N_{3}\left\langle r^{4}\right\rangle^{3}
\end{aligned}
$$

\footnotetext{
5 Here, recentering is an event-by-event shift to the center of mass.

6 As pointed out in Ref. [35], recentering can be effectively included by changing $N \rightarrow N-1$. In this case both MC and our analytical calculations agree very well for all $N$.
} 


$$
\begin{aligned}
& +20 N_{4}\left\langle r^{2}\right\rangle^{3}\left\langle r^{6}\right\rangle+60 N_{3}\left\langle r^{2}\right\rangle\left\langle r^{4}\right\rangle\left\langle r^{6}\right\rangle+10 N_{2}\left\langle r^{6}\right\rangle^{2}+15 N_{3}\left\langle r^{2}\right\rangle^{2}\left\langle r^{8}\right\rangle \\
& +15 N_{2}\left\langle r^{4}\right\rangle\left\langle r^{8}\right\rangle+6 N_{2}\left\langle r^{2}\right\rangle\left\langle r^{10}\right\rangle+N\left\langle r^{12}\right\rangle
\end{aligned}
$$

and finally for $\left\langle\epsilon_{2}^{8}\right\rangle$

$$
\begin{aligned}
\left\langle\epsilon_{2}^{8}\right\rangle_{\text {num }}= & 24 N_{4}\left\langle r^{4}\right\rangle^{4}+72 N_{3}\left\langle r^{4}\right\rangle^{2}\left\langle r^{8}\right\rangle+18 N_{2}\left\langle r^{8}\right\rangle^{2}+16 N_{2}\left\langle r^{4}\right\rangle\left\langle r^{12}\right\rangle+N\left\langle r^{16}\right\rangle \\
\left\langle\epsilon_{2}^{8}\right\rangle_{\text {deno }}= & N_{8}\left\langle r^{2}\right\rangle^{8}+28 N_{7}\left\langle r^{2}\right\rangle^{6}\left\langle r^{4}\right\rangle+210 N_{6}\left\langle r^{2}\right\rangle^{4}\left\langle r^{4}\right\rangle^{2}+420 N_{5}\left\langle r^{2}\right\rangle^{2}\left\langle r^{4}\right\rangle^{3} \\
& +105 N_{4}\left\langle r^{4}\right\rangle^{4}+56 N_{6}\left\langle r^{2}\right\rangle^{5}\left\langle r^{6}\right\rangle+560 N_{5}\left\langle r^{2}\right\rangle^{3}\left\langle r^{4}\right\rangle\left\langle r^{6}\right\rangle+840 N_{4}\left\langle r^{2}\right\rangle\left\langle r^{4}\right\rangle^{2}\left\langle r^{6}\right\rangle \\
& +280 N_{4}\left\langle r^{2}\right\rangle^{2}\left\langle r^{6}\right\rangle^{2}+280 N_{3}\left\langle r^{4}\right\rangle\left\langle r^{6}\right\rangle^{2}+70 N_{5}\left\langle r^{2}\right\rangle^{4}\left\langle r^{8}\right\rangle+420 N_{4}\left\langle r^{2}\right\rangle^{2}\left\langle r^{4}\right\rangle\left\langle r^{8}\right\rangle \\
& +210 N_{3}\left\langle r^{4}\right\rangle^{2}\left\langle r^{8}\right\rangle+280 N_{3}\left\langle r^{2}\right\rangle\left\langle r^{6}\right\rangle\left\langle r^{8}\right\rangle+35 N_{2}\left\langle r^{8}\right\rangle^{2}+56 N_{4}\left\langle r^{2}\right\rangle^{3}\left\langle r^{10}\right\rangle \\
& +168 N_{3}\left\langle r^{2}\right\rangle\left\langle r^{4}\right\rangle\left\langle r^{10}\right\rangle+56 N_{2}\left\langle r^{6}\right\rangle\left\langle r^{10}\right\rangle+28 N_{3}\left\langle r^{2}\right\rangle^{2}\left\langle r^{12}\right\rangle+28 N_{2}\left\langle r^{4}\right\rangle\left\langle r^{12}\right\rangle \\
& +8 N_{2}\left\langle r^{2}\right\rangle\left\langle r^{14}\right\rangle+N\left\langle r^{16}\right\rangle .
\end{aligned}
$$

Using above equations we performed calculations for various functions $P_{r}(r)$, and found that Eq. (21) is always satisfied with good accuracy. ${ }^{7}$

\section{Concluding remarks}

In conclusion, we find an exact formula for $\left\langle\epsilon_{2}^{2 n}\right\rangle$ for $N$ independent points sampled according to a general distribution $P(r, \phi)$. We restrict ourselves to a systems dominated by fluctuations, so that on average this system is azimuthally symmetric. This should apply to $\mathrm{p}+\mathrm{A}$ collisions at the LHC. We also derived explicit relations for the cumulants, $\epsilon_{2}\{2 n\}$, for a Gaussian distribution. Finally, we analytically verified the recently observed numerical relation $\epsilon_{2}\{2\}>\epsilon_{2}\{4\} \simeq \epsilon_{2}\{6\} \simeq \epsilon_{2}\{8\}$ between cumulants in $\mathrm{p}+$ A collisions.

\section{Acknowledgements}

We thank R. Pisarski for valuable comments. A.B. was supported through the RIKENBNL Research Center, by the Ministry of Science and Higher Education (MNiSW), by founding from the Foundation for Polish Science, and by the National Science Centre, Grant No. DEC-2014/15/B/ST2/00175, and in part by DEC-2013/09/B/ST2/00497. V.S. acknowledges support and hospitality of RIKEN-BNL Research Center, where this work was initiated.

\section{References}

[1] V. Khachatryan, et al., CMS Collaboration, J. High Energy Phys. 1009 (2010) 091, arXiv:1009.4122 [hep-ex].

[2] S. Chatrchyan, et al., CMS Collaboration, Phys. Lett. B 718 (2013) 795, arXiv:1210.5482 [nucl-ex].

[3] B. Abelev, et al., ALICE Collaboration, Phys. Lett. B 719 (2013) 29, arXiv:1212.2001.

[4] G. Aad, et al., ATLAS Collaboration, Phys. Rev. Lett. 110 (2013) 182302, arXiv:1212.5198 [hep-ex].

[5] A. Adare, et al., PHENIX Collaboration, Phys. Rev. Lett. 111 (2013) 212301, arXiv:1303.1794 [nucl-ex].

[6] A. Adare, et al., arXiv:1507.06273 [nucl-ex].

[7] J. Adams, et al., STAR Collaboration, J. Phys. G 32 (2006) L37, arXiv:nucl-ex/0509030.

\footnotetext{
7 In particular, we considered $P_{r}(r) \sim e^{-r^{k} / \sigma^{k}}$ with $\left\langle r^{m}\right\rangle=\sigma^{m} \Gamma\left(\frac{2+m}{k}\right) / \Gamma\left(\frac{2}{k}\right)$ for various values of $k$, and $P_{r}(r) \sim$ $\Theta\left(r_{0}-r\right)$ with $\left\langle r^{m}\right\rangle=2 r_{0}^{m} /(2+m)$.
} 
[8] W. Florkowski, Phenomenology of Ultra-Relativistic Heavy-Ion Collisions, World Scientific Publishing Company, Singapore, 2010.

[9] H. Song, U.W. Heinz, J. Phys. G 36 (2009) 064033, arXiv:0812.4274 [nucl-th].

[10] D.T. Son, A.O. Starinets, Annu. Rev. Nucl. Part. Sci. 57 (2007) 95, arXiv:0704.0240 [hep-th].

[11] F. Gelis, E. Iancu, J. Jalilian-Marian, R. Venugopalan, Annu. Rev. Nucl. Part. Sci. 60 (2010) 463, arXiv:1002.0333 [hep-ph].

[12] K. Dusling, R. Venugopalan, Phys. Rev. D 87 (2013) 094034, arXiv:1302.7018 [hep-ph].

[13] Y.V. Kovchegov, D.E. Wertepny, Nucl. Phys. A 906 (2013) 50, arXiv:1212.1195.

[14] A. Kovner, M. Lublinsky, Int. J. Mod. Phys. E 22 (2013) 1330001, arXiv:1211.1928 [hep-ph].

[15] B. Schenke, S. Schlichting, R. Venugopalan, Phys. Lett. B 747 (2015) 76, arXiv:1502.01331 [hep-ph].

[16] A. Dumitru, A.V. Giannini, V. Skokov, arXiv:1503.03897 [hep-ph]; V. Skokov, Phys. Rev. D 91 (5) (2015) 054014;

A. Dumitru, V. Skokov, Phys. Rev. D 91 (7) (2015) 074006;

A. Dumitru, L. McLerran, V. Skokov, Phys. Lett. B 743 (2015) 134.

[17] P. Bozek, Phys. Rev. C 85 (2012) 014911, arXiv:1112.0915 [hep-ph].

[18] A. Bzdak, B. Schenke, P. Tribedy, R. Venugopalan, Phys. Rev. C 87 (2013) 064906, arXiv:1304.3403 [nucl-th].

[19] P. Bozek, W. Broniowski, G. Torrieri, Phys. Rev. Lett. 111 (2013) 172303, arXiv:1307.5060 [nucl-th].

[20] G.Y. Qin, B. Mueller, Phys. Rev. C 89 (4) (2014) 044902, arXiv:1306.3439 [nucl-th].

[21] E. Shuryak, I. Zahed, Phys. Rev. C 88 (2013) 044915, arXiv:1301.4470 [hep-ph].

[22] I. Kozlov, M. Luzum, G. Denicol, S. Jeon, C. Gale, arXiv:1405.3976 [nucl-th].

[23] T. Kalaydzhyan, E. Shuryak, Phys. Rev. C 90 (1) (2014) 014901, arXiv:1404.1888 [hep-ph].

[24] I. Iatrakis, A. Ramamurti, E. Shuryak, Phys. Rev. D 92 (1) (2015) 014011, arXiv:1503.04759 [hep-ph].

[25] Z.W. Lin, C.M. Ko, B.A. Li, B. Zhang, S. Pal, Phys. Rev. C 72 (2005) 064901, arXiv:nucl-th/0411110.

[26] G.L. Ma, A. Bzdak, Phys. Lett. B 739 (2014) 209, arXiv:1404.4129 [hep-ph].

[27] A. Bzdak, G.L. Ma, Phys. Rev. Lett. 113 (25) (2014) 252301, arXiv:1406.2804 [hep-ph].

[28] J.D.O. Koop, A. Adare, D. McGlinchey, J.L. Nagle, arXiv:1501.06880 [nucl-ex].

[29] L. He, T. Edmonds, Z.W. Lin, F. Liu, D. Molnar, F. Wang, arXiv:1502.05572 [nucl-th].

[30] A. Bzdak, V. Skokov, Phys. Rev. Lett. 111 (2013) 182301, arXiv:1307.6168 [hep-ph].

[31] P. Bozek, A. Bzdak, V. Skokov, Phys. Lett. B 728 (2014) 662, arXiv:1309.7358 [hep-ph].

[32] C.E. Coleman-Smith, B. Mueller, Phys. Rev. D 89 (2) (2014) 025019, arXiv:1307.5911 [hep-ph].

[33] G. Basar, D. Teaney, Phys. Rev. C 90 (5) (2014) 054903, arXiv:1312.6770 [nucl-th].

[34] A. Bzdak, P. Bozek, L. McLerran, Nucl. Phys. A 927 (2014) 15, arXiv:1311.7325 [hep-ph].

[35] L. Yan, J.Y. Ollitrault, Phys. Rev. Lett. 112 (2014) 082301, arXiv:1312.6555 [nucl-th].

[36] B. Alver, et al., PHOBOS Collaboration, Phys. Rev. Lett. 98 (2007) 242302, arXiv:nucl-ex/0610037.

[37] D. Teaney, L. Yan, Phys. Rev. C 83 (2011) 064904, arXiv:1010.1876 [nucl-th].

[38] R.S. Bhalerao, M. Luzum, J.-Y. Ollitrault, Phys. Rev. C 84 (2011) 054901, arXiv:1107.5485 [nucl-th].

[39] B. Alver, et al., Phys. Rev. C 77 (2008) 014906, arXiv:0711.3724 [nucl-ex].

[40] N. Borghini, P.M. Dinh, J.-Y. Ollitrault, Phys. Rev. C 64 (2001) 054901, arXiv:nucl-th/0105040.

[41] M. Miller, R. Snellings, arXiv:nucl-ex/0312008. 